\section{Utilização de serviços odontológicos por crianças de 0 a 5 anos de idade no Município de Canela, Rio Grande do Sul, Brasil}

\author{
Use of dental services by preschool children in \\ Canela, Rio Grande do Sul State, Brazil
}

\begin{abstract}
The aim of this study was to assess the use of dental services and age at first dental visit in preschool children in Canela, Rio Grande do Sul State, Brazil. A representative sample of underfive children was surveyed on National Children's Vaccination Day. Children's parents completed questionnaires containing socio-demographic data and age at first dental visit. Data were analyzed using multiple logistic regression. 192 children were examined. $13.3 \%$ of the sample had already visited the dentist at least once, but only 4.3\% had their first dental visit by one year of age. The number of children who had already visited a dentist increased with age. Girls showed higher odds of having visited a dentist $(O R=1.46$; 95\%CI: 1.01-2.1). Public health strategies are needed to determine the effectiveness of health promotion and improve the use of dental services by preschool children.
\end{abstract}

Child; Dental Health Services; Health Services Accessibility
Paulo Floriani Kramer ${ }^{1}$

Thiago Machado Ardenghi 2

Simone Ferreira 1

Laura de Almeida Fischer 1

Luciana Cardoso ${ }^{1}$

Carlos Alberto Feldens 1

A odontologia tem evoluído de um enfoque curativo dos problemas bucais para um olhar mais dinâmico dos determinantes do processo saúdedoença. Repercussões nessa mudança de atitude fazem com que medidas e estratégias de atenção odontológica sejam preconizadas precocemente para evitar e/ou diminuir as seqüelas dos principais problemas que afetam a saúde bucal da população. A atenção odontológica em idades precoces torna-se, dessa forma, uma importante estratégia na redução das seqüelas das doenças bucais mais prevalentes e do custo do tratamento destas 1,2 .

Tem-se preconizado que a idade ideal para a primeira consulta odontológica é entre 6 e 12 meses, na época de erupção do primeiro dente decíduo 3,4 . Tal indicação se justifica pela importância do atendimento odontológico em idades precoces, que tem o intuito de facilitar o estabelecimento de hábitos saudáveis, além de servir como uma oportunidade fundamental para avaliação do desenvolvimento crânio-facial e todos os fatores de risco comuns a que uma criança possa estar exposta 5,6 .

Entretanto, no Brasil, o acesso a serviços odontológicos é limitado e desigual. Com base no último levantamento epidemiológico em saúde bucal realizado pelo Ministério da Saúde, aproximadamente $14 \%$ dos jovens entre 15 e 19 anos nunca foram ao cirurgião-dentista. O mes- 
mo documento relata ainda desigualdades regionais marcantes: por exemplo, enquanto, na Região Sul, menos de $6 \%$ dos jovens dessa faixa etária relataram nunca ter ido ao dentista, no Nordeste a porcentagem chega a quase $22 \% 7$.

De acordo com dados da Pesquisa Nacional porAmostra de Domicílios (PNAD), embora exista baixa taxa de procura de serviços odontológicos em pré-escolares, houve uma redução na porcentagem de crianças menores de quatro anos que nunca haviam procurado o cirurgião-dentista entre 1998 e 2003 (de 85,7\% para 81,9\%, respectivamente) 8 . Todavia, estudos nacionais a respeito da idade em que a criança realizou a primeira consulta odontológica e da taxa de utilização desses serviços em pré-escolares ainda são escassos, principalmente tendo como característica a análise de amostras significativas da população.

Essas pesquisas são importantes porque permitem, a partir de uma necessidade constatada, a organização de políticas públicas de promoção de saúde com intuito de informar e conduzir a população ao atendimento odontológico precoce. Dessa forma, o objetivo do presente estudo foi verificar a prevalência de crianças em idade préescolar que já consultaram o cirurgião-dentista e a idade em que a primeira consulta odontológica foi realizada, em uma amostra representativa de pré-escolares do Município de Canela, Rio Grande do Sul, Brasil.

\section{Material e métodos}

O Município de Canela tem uma população de 33.625 habitantes, sendo a população entre 0 e 4 anos de idade estimada em 3.361 crianças e entre 5 e 6 anos, em 1.251 9. Dados da Secretaria Municipal de Saúde (SMS-Canela) revelam uma cobertura vacinal acima de $90 \%$ da população de 0 a 5 anos de idade.

Durante a Campanha Nacional de Multivacinação realizada na cidade, no ano de 2003, desenvolveu-se um estudo transversal abrangendo aspectos relacionados à saúde bucal de crianças entre 0 e 5 anos de idade. A amostra desse estudo foi obtida por um processo de conglomerado em um único estágio.

O tamanho da amostra necessária para verificar a freqüência de crianças que já haviam consultado o cirurgião-dentista, considerando um poder de $80 \%$, nível de confiança de $95 \%$ e margem de erro de $3 \%$, seria de 797 crianças. Contudo, o projeto verificou outros desfechos, exigindo tamanho de amostra maior. Ao se acrescentarem 5\% para possíveis perdas por erro de preenchimento, totalizou-se um tamanho amostral requerido de 1.042 crianças.
A SMS-Canela estabelecera 12 postos de vacinação, estimando um total de 3.477 crianças vacinadas. Considerando a previsão da Secretaria de que haveria um número semelhante de crianças sendo vacinadas em cada posto e assumindo que a perda de homogeneidade não seria significativa (todos os postos eram localizados na zona urbana), foram sorteados aleatoriamente seis postos de vacinação para que se atingisse o tamanho amostral desejado. Em cada posto, foram examinadas todas as crianças cujos responsáveis concordassem em participar do estudo.

\section{Coleta dos dados}

Os dados foram coletados por seis equipes de trabalho formadas por um examinador, um anotador e auxiliares do próprio posto de saúde, distribuídos nos seis pontos de vacinação. Os participantes do estudo foram selecionados de todas as crianças que procuraram atendimento nas unidades de vacinação. As crianças eram examinadas nas unidades básicas de saúde para a avaliação dos diferentes desfechos considerados no projeto.

Um questionário contendo informações relativas a consulta odontológica (se já havia ido ao dentista e idade em anos da primeira consulta) e dados demográficos do paciente (idade e sexo) era respondido pelos responsáveis. Todos os dados foram coletados em um único dia por examinadores previamente treinados.

O instrumento de coleta de dados foi definido após avaliação da validade e reprodutibilidade do questionário, verificada por estudo-piloto realizado um mês antes da coleta dos dados, com aproximadamente $10 \%$ da amostra requerida.

Todos os responsáveis assinaram um Termo de Consentimento Livre e Esclarecido, permitindo sua participação na pesquisa.

Este estudo foi aprovado pelo Comitê de Ética em Pesquisa da Universidade Luterana do Brasil (ULBRA), seguindo todos os princípios éticos, uma vez que os resultados da presente pesquisa envolveram seres humanos.

\section{Análise dos dados}

Os dados foram analisados estatisticamente utilizando-se o programa SPSS versão 11.0 (SPSS Inc., Chicago, Estados Unidos). Foram descritas as freqüências simples e relativas de consulta prévia ao cirurgião-dentista nas categorias das variáveis de exposição avaliadas. Para verificar a associação entre idade da criança e o desfecho foi realizado teste qui-quadrado $\left(\chi^{2}\right)$ para tendência linear. Para verificar o efeito independente das variáveis sexo e faixa etária sobre o desfecho (se 
já havia ou não consultado o cirurgião-dentista), foi realizada a análise de regressão logística, sendo calculadas as razões de chances simples e ajustadas (sexo ajustado para idade e vice-versa) e intervalos de confiança de 95\% (IC95\%).

\section{Resultados}

Um total de 1.092 crianças participou do estudo, sendo 551 (50,5\%) meninos e 541 (49,5\%) meninas. A taxa de resposta à participação foi de 98\% de todas as crianças convidadas para fazer parte do estudo. As principais razões para não participação foram recusa por parte da criança e impossibilidade de responder a questionário por parte dos responsáveis.

De acordo com a Tabela 1, pode-se verificar que 145 crianças, correspondendo a apenas 13,3\% (IC95\%: 11,3-15,3) da amostra, já haviam realizado algum tipo de consulta odontológica. Observou-se que, à medida que aumentava a idade, aumentava a freqüência de crianças que tinham consultado o cirurgião-dentista $\left(\chi^{2}\right.$ para tendência linear; $\mathrm{p}<0,001)$. Das crianças menores de 2 anos, apenas 4,3\% (12) já haviam consultado o cirurgião-dentista; das crianças entre 2 e 3 anos, o percentual foi de $11,2 \%$ (60) e entre aquelas maiores de 3 anos de idade o percentual foi de $26,2 \%$ (74) (Tabela 1).

Quando os resultados foram discriminados de acordo com as diferentes faixas etárias, pôde-se observar que houve associação entre idade e consulta ao cirurgião-dentista (Tabela 2; p < 0,005). Crianças entre 2 e 3 anos apresentaram quase três vezes mais chance de terem ido ao consultório odontológico do que as de 0 e 1 ano de idade (OR = 2,79; IC95\%: 1,47-5,27); da mes- ma forma, as de 4 e 5 anos apresentaram aproximadamente oito vezes mais chance do que as crianças menores de 2 anos (OR = 7,96; IC95\%: 4,21-15,05).

Houve associação entre gênero e o número de crianças que já haviam realizado consulta odontológica (Tabela 2). Crianças do sexo feminino apresentavam maior chance de ter ido ao cirurgião-dentista quando comparadas àquelas do sexo masculino (OR = 1,46; IC95\%: 1,01-2,1).

\section{Discussão}

A principal razão da realização deste estudo refere-se ao fato de que estes resultados podem servir de base para a implementação de políticas públicas de saúde com um enfoque voltado para o incentivo à procura de atendimento odontológico em idade precoce por parte da população. Isso traria um retorno direto à população estudada, na medida em que permitiria uma redução nos custos com tratamento e atendimento das seqüelas dos principais problemas bucais que afetam pré-escolares 10 .

Dados em nível nacional revelam alta proporção de pré-escolares que nunca procuraram atendimento odontológico ${ }^{8}$. De acordo com a última PNAD, realizada em 2003, aproximadamente $80 \%$ de crianças menores de cinco anos nunca haviam realizado uma consulta odontológica ${ }^{9}$, o que corrobora os resultados do presente estudo, segundo o qual apenas uma pequena parte dos pré-escolares do Município de Canela (13,3\%) já havia consultado o cirurgião-dentista (Tabela 1). Essa porcentagem foi menor que a encontrada em outros estudos internacionais 11,12,13. Em 2000, apenas 21,1\% das crianças ame-

Freqüências simples e percentuais e intervalos de confiança de $95 \%$ de crianças que utilizaram serviços odontológicos de acordo com a idade. Canela, Rio Grande do Sul, Brasil.

\begin{tabular}{lcccc}
\hline Idade (anos) & $\mathbf{N}$ & \multicolumn{2}{c}{$\begin{array}{c}\text { Realizaram consulta odontológica } \\
\%\end{array}$} & IC95\% \\
\hline$<1$ & & $\mathbf{n}$ & 8,7 & $0,6-16,8$ \\
1 & 46 & 4 & 3,4 & $1,1-5,7$ \\
2 & 232 & 8 & 9,1 & $5,7-12,5$ \\
3 & 265 & 24 & 13,2 & $9,2-17,2$ \\
4 & 272 & 36 & 25,9 & $20,6-31,2$ \\
5 & 255 & 66 & 31,8 & $12,4-51,2$ \\
Total & 22 & 7 & 13,3 & $11,3-15,3$ \\
\hline
\end{tabular}

* Teste qui-quadrado para tendência linear. 
Associação entre a consulta odontológica, o gênero e a faixa etária. Canela, Rio Grande do Sul, Brasil.

\begin{tabular}{|c|c|c|c|c|c|c|c|c|c|}
\hline \multirow[t]{2}{*}{ Variáveis } & \multirow[t]{2}{*}{ Total } & \multicolumn{2}{|c|}{ Consulta odontológica } & \multicolumn{3}{|c|}{ Modelo bruto } & \multicolumn{3}{|c|}{ Modelo ajustado * } \\
\hline & & $\mathrm{n}$ & $\%$ & $\begin{array}{l}\text { Razão de } \\
\text { chance }\end{array}$ & IC95\% & $\mathrm{p}$ & $\begin{array}{c}\text { Razão de } \\
\text { chance }\end{array}$ & IC95\% & $p$ \\
\hline \multicolumn{10}{|l|}{ Gênero } \\
\hline Masculino & 551 & 61 & 11,0 & 1,00 & - & & 1,00 & - & \\
\hline Feminino & 541 & 84 & 15,5 & 1,49 & $1,05-2,12$ & 0,026 & 1,46 & $1,01-2,10$ & 0,040 \\
\hline \multicolumn{10}{|l|}{ Idade (anos) } \\
\hline$<2$ & 278 & 12 & 4,3 & 1,00 & - & & 1,00 & - & \\
\hline $2-3$ & 537 & 60 & 11,2 & 2,79 & $1,47-5,27$ & 0,002 & 2,74 & $1,45-5,19$ & 0,002 \\
\hline$\geq 4$ & 277 & 73 & 26,4 & 7,96 & $4,21-15,05$ & 0,000 & 7,91 & $4,18-14,95$ & 0,000 \\
\hline
\end{tabular}

* Gênero ajustado para idade; idade ajustada para gênero.

ricanas menores de seis anos de idade já haviam procurado atendimento odontológico 13. Em outro estudo conduzido com uma coorte de crianças americanas acompanhadas do nascimento até os três anos de idade, pôde-se constatar que apenas $2 \%$ destas haviam recebido consulta odontológica antes de um ano de idade e $31 \%$, aos três anos 12 .

Essa baixa procura por atendimento odontológico relatada no presente estudo torna-se preocupante, pois revela a falta de uma política de incentivo e apoio às medidas de atenção odontológica precoce, tanto por parte da população, como por parte do setor público.

A baixa prevalência de crianças que já haviam procurado atendimento odontológico (Tabela 1) poderia, ainda, ser explicada por diversos fatores, embora poucos estudos tenham sido realizados na literatura para verificar os motivos que influenciam a procura por atendimento odontológico em crianças de pequena idade. Em um desses estudos, Edelstein et al. 11 relataram que os baixos níveis sócio-econômicos estavam positivamente associados à menor procura por atendimento odontológico. Ou seja, até em setores públicos a utilização de atendimentos precoces é desigualmente distribuída na população, reforçando ainda mais a necessidade de medidas de atenção voltadas à diminuição das desigualdades em saúde, mesmo no que se refere à procura de atendimento odontológico precoce.

A associação observada entre idade e a porcentagem de crianças que já havia realizado consulta odontológica está de acordo com os achados de Slayton et al. ${ }^{12}$, os quais verificaram que há um aumento de $2 \%$ a $26 \%$ no número de crianças que procuram os serviços odontológicos entre as idades de 1 a 3 anos respectivamente.
Porém, seja no estudo descrito, seja no presente trabalho, as razões para esse aumento de acordo com a idade e os motivos que levaram as crianças a procurarem o atendimento não foram completamente explorados. O aumento poderia ser causado tanto por uma necessidade percebida de atendimento preventivo por parte dos responsáveis, como por uma necessidade de intervenção em seqüelas de problemas bucais que se tornam mais prevalentes de acordo com o aumento de idade. Novos estudos deverão ser realizados a fim de elucidar melhor essa questão, tendo como base, principalmente, amostras populacionais representativas que permitam a extrapolação e comparação dos resultados com outros achados da literatura.

O fato de que crianças do sexo feminino apresentaram uma maior chance de ter ido ao cirurgião-dentista quando comparadas àquelas do sexo masculino (OR = 1,46; IC95\%:1,01-2,1) já foi anteriormente observado 8 . Em estudo realizado com base em dados provenientes da PNAD-2003, os autores constataram que a chance de nunca ter visitado o dentista foi $20 \%$ maior para os homens em comparação com mulheres, independente de faixa etária.

Diferenças entre gênero em termos de procura e acesso a serviços de saúde têm sido documentadas na literatura; as mulheres, de um modo geral, procuram mais atendimento que os homens 14. Embora não haja um consenso a respeito da real influência do gênero na procura por atendimento de saúde, tem-se demonstrado que fatores psicológicos relacionados à auto-avaliação referida de saúde podem, de certa forma, explicar tais diferenças 15. Mulheres tendem a apresentar maior interesse por sua saúde, em virtude de terem maior autopercepção de saúde 
do que os homens, o que faz com que elas procurem mais os serviços médico e odontológico 8,14 . Entretanto, há de se considerar que, no presente estudo, nenhum indicador de necessidade percebida foi utilizado. Além disso, a população investigada foi de crianças pré-escolares, e a procura por atendimento é dependente das decisões dos responsáveis. Assim, ainda que seja relatada uma maior percepção em saúde e impacto das injúrias nas atividades diárias em crianças do sexo feminino 16 , novos estudos que relacionem a utilização de tais indicadores e que empreguem os responsáveis das crianças como proxy são necessários para elucidar o real mecanismo que explicaria as diferenças encontradas em termos de procura de atendimento odontológico e relação entre os gêneros nesta faixa etária.

Das crianças que já haviam procurado o cirurgião-dentista, apenas uma pequena porcentagem realizou a consulta até o primeiro ano de vida; a grande maioria das crianças teve a primeira consulta entre 2 e 3 anos. Embora a primeira consulta odontológica seja uma oportunidade de orientar crianças e responsáveis, promovendo atitudes saudáveis, a idade ideal para primeira consulta odontológica, principalmente em crianças sem sinais ou seqüelas de problemas bucais, ainda gera muitas discussões na literatura, seja por parte dos responsáveis, seja por parte dos profissionais. Zuanon et al. 17, durante a Campanha de Vacinação na cidade de Araraquara, São Paulo, Brasil, mostraram que $43 \%$ das mães acharam que seus filhos deveriam ser levados para a primeira consulta odontológica até o primeiro ano de vida; $44 \%$ achavam que essa consulta deveria ocorrer entre 1 e 3 anos, $17 \%$ acreditavam que deveria ser a partir dos 3 anos e $6 \%$ diziam que só deveriam levar a criança ao dentista em situações de emergência. Em outro estudo realizado com gestantes de Campinas, São Paulo, verificou-se que quase metade das entrevistadas relatou não ter conhecimento de qual seria a idade ideal para a primeira consulta odontológica 18.

A primeira consulta odontológica deveria servir como uma estratégia para reduzir a prevalência e as seqüelas de problemas bucais, bem como os custos com serviços de intervenção em saúde 2,10 , pois crianças que visitaram o cirurgião-dentista até o primeiro ano de vida, apresentam menores chances de receberem tratamento odontológico emergencial e de fazerem visitas de urgência ao longo da infância 10, reduzindo, assim, os custos despendidos em saúde pública. Entretanto, no Brasil, ainda não há estudos que comprovem a relação custo-benefício de qualquer protocolo de atendimento em idade precoce. A realização de tais estudos auxiliaria sensivelmente a tomada de decisões e estratégias de atenção que permitissem uma maior alocação de recursos financeiros em termos de saúde pública.

Há de se considerar, ainda, que os resultados do presente estudo precisam ser vistos com cautela. Os dados aqui expressos devem ser considerados para esta população específica, havendo a necessidade de mais estudos considerando diferentes populações, com diferentes níveis sócio-econômicos. Mesmo que tenha sido realizado um estudo com uma amostra representativa, vieses poderiam ocorrer principalmente no que se refere à recordação da idade em que a criança procurou o atendimento pela primeira vez. Além disso, no presente estudo não foram avaliados dados sócio-econômicos que poderiam influenciar a decisão de procura por atendimento odontológico, como descrito previamente por outros estudos de base nacional 8,14. O efeito de tais variáveis de confusão necessita ser mais bem elucidado na referida população para melhor inferência desses resultados.

Houve diferenças entre tamanhos amostrais para crianças de um ano e para crianças de cinco anos em relação às demais faixas etárias (46 e 22 respectivamente - Tabela 1). Esse fato refletiu a população que procurou os postos de vacinação, não tendo ocorrido seleção diferencial de crianças das outras faixas etárias. Durante o primeiro ano de vida, as crianças apresentam diferentes vulnerabilidades e recebem atenção médica (principalmente atenção primária) com maior freqüência. As características e recomendações especiais nestas faixas etárias podem ter determinado uma menor freqüência de vacinação. Em adição, a menor freqüência de crianças de cinco anos de idade deve decorrer da menor motivação dos responsáveis por crianças nesta idade limítrofe. É possível que este fato tenha repercutido nos resultados encontrados no estudo, principalmente no que se refere às crianças de um ano de idade. Como foi muito menor o número de crianças examinadas nesta faixa etária, a estimativa do desfecho não apresentou a precisão observada nas demais idades até quatro anos, o que pode ser observado pela amplitude maior do intervalo de confiança. $O$ fato de estas crianças estarem sub-representadas neste estudo pode ter superestimado a estimativa global (0 a 5 anos) de consulta ao cirurgiãodentista, mas não interferiu nas prevalências observadas nas demais idades, um dos escopos do presente estudo.

O processo amostral foi realizado por conglomerados em um único estágio (unidades de saúde). Em face das características da coleta de dados - realizada em um único dia de vacina- 
ção -, não foi possível determinar previamente o coeficiente de correlação intraclasses dos conglomerados. Dessa forma, não foi considerado a priori o efeito do delineamento para o cálculo amostral, o que diminui a precisão dos resultados obtidos. No entanto, é possível que o erro amostral decorrente não tenha afetado significativamente os resultados, uma vez que o número de crianças efetivamente examinadas foi $37 \%$ superior ao número amostral requerido para este desfecho. Ademais, o erro amostral parece não inviabilizar os resultados expostos, na medida em que revela um panorama geral da realidade de um município no que se refere à utilização dos serviços odontológicos, além de prover dados importantes para formulação de medidas públicas com enfoque na redução das desigualdades em saúde e, sobretudo, no incentivo à utilização desses serviços 19 .

\section{Conclusão}

Pode-se concluir que houve uma baixa taxa de uso de serviços odontológicos na população estudada, sendo necessário o estabelecimento de políticas públicas de promoção de saúde e reorientação de serviços que facilitem a utilização dos serviços odontológicos e possibilitem o atendimento às crianças de pouca idade.

\section{Resumo}

O objetivo deste estudo foi verificar a utilização de serviços odontológicos e idade da primeira visita odontológica em pré-escolares do Município de Canela, Rio Grande do Sul, Brasil. Um levantamento epidemiológico em crianças menores de cinco anos de idade foi realizado durante a Campanha Nacional de Multivacinação Infantil no município. Um questionário contendo informações a respeito da idade da primeira consulta odontológica e características demográficas da criança foi respondido pelos responsáveis. Os dados foram analisados utilizando-se modelo de regressão logística. Um total de 1.092 crianças foram examinadas. Do total da amostra, 13,3\% já haviam consultado o cirurgião-dentista, e somente 4,3\% realizaram algum tipo de consulta odontológica até o primeiro ano de vida. O número de crianças que já haviam recebido atendimento odontológico aumentou com a idade. Crianças do sexo feminino apresentaram maior chance de ter recebido atendimento odontológico do que as do sexo masculino (OR = 1,46; IC95\%: 1,01-2,1). Conclui-se que é necessário o estabelecimento de políticas públicas de promoção de saúde e reorientação de serviços que facilitem a utilização dos serviços odontológicos e possibilitem o atendimento às crianças de pouca idade.

Criança; Serviços de Saúde Bucal; Acesso aos Serviços de Saúde

\section{Colaboradores}

P. F. Kramer coordenou o projeto e redigiu o artigo. T. M. Ardenghi redigiu o artigo e colaborou na análise estatística. S. Ferreira colaborou na coleta de dados e na redação do artigo. L. A. Fischer coletou os dados e colaborou na redação do artigo. C. A. Feldens realizou as análises estatísticas e colaborou na redação do artigo. L. Cardoso colaborou na coleta dos dados. 


\section{Referências}

1. Ismail AI, Nainar SM, Sohn W. Children's first dental visit: attitudes and practices of pediatricians and family physicians. Pediatr Dent 2003; 25:42530 .

2. Savage MF, Lee JY, Kotch JB, Vann Jr. WF. Early preventive dental visits: effects on subsequent utilization and costs. Pediatrics 2004; 114:418-23.

3. Hashim-Nainar SM, Straffon LH. Targeting of the year one dental visit for United States children. Int J Paediatr Dent 2003; 13:258-63.

4. Rayner JA. The first dental visit: a UK viewpoint. Int J Paediatr Dent 2003; 13:269.

5. Poulsen S. The child's first dental visit. Int J Paediatr Dent 2003; 13: 264-265.

6. Bönecker MJ, Sheiham A. Promovendo saúde bucal na infância e adolescência: conhecimentos e práticas. São Paulo: Editora Santos; 2004.

7. Coordenação Nacional de Saúde Bucal, Ministério da Saúde. Condições de saúde bucal da população brasileira. Resultados principais. http://dtr2001. saude.gov.br/editora/produtos/livros/zip/04_ 0347_M.zip (acessado em 10/Jan/2005).

8. Pinheiro RS, Torres TZ. Uso de serviços odontológicos entre os Estados do Brasil. Ciênc Saúde Coletiva 2006; 11:999-1010.

9. Instituto Brasileiro de Geografia e Estatística. Estimativas municipais e revisão 2004 da projeção da população. http://www.ibge.gov.br/home/ presidencia/noticias/27072004estimativas2004. shtm (acessado em 22/Set/2004).

10. Lee JY, Bouwens TJ, Savage MF, Vann Jr. WF. Examining the cost-effectiveness of early dental visits. Pediatr Dent 2006; 28:102-5.
11. Edelstein BL. Access to dental care for Head Start enrollees. J Public Health Dent 2000; 60:221-9.

12. Slayton RL, Warren JJ, Levy SM, Kanellis MJ, Islam M. Frequency of reported dental visits and professional fluoride applications in a cohort of children followed from birth to age 3 years. Pediatr Dent 2002; 24:64-8.

13. Siegal MD, Marx ML, Cole SL. Parent or caregiver staff, and dentist perspectives on access to dental care issues for head start children in Ohio. Am J Public Health 2005; 8:1352-9.

14. Pinheiro RS, Viacava F, Travassos C, Brito AS. Gênero, morbidade, acesso e utilização de serviços de saúde no Brasil. Ciênc Saúde Coletiva 2002; 7:687-707.

15. Verbrugge LM. The twain meet: empirical explanations of sex differences in health and mortality. J Health Soc Behav 1989; 30:282-304.

16. Jiang H, Petersen PE, Peng B, Tai B, Bian Z. Selfassessed dental health, oral health practices, and general health behaviors in Chinese urban adolescents. Acta Odontol Scand 2005; 63:343-52.

17. Zuanon ACC, Motisuki C, Bordin MM, Zuim K. Quando levar a criança para a primeira visita ao dentista? J Bras Odontopediatr Odontol Bebê 2001; 4:321-4.

18. Politano GT, Pellegrinetti MB, Echeverria SR, Imparato JCP. Avaliação da informação das mães sobre cuidados bucais com o bebê. JBP Rev Iberoam Odontopediatr Odontol Bebê 2004; 36:138-48.

19. Fernandes SF, Peres MA. Associação entre atenção básica em saúde bucal e indicadores sócioeconômicos municipais. Rev Saúde Pública 2005; 39:930-6.

Recebido em 16/Abr/2006

Versão final reapresentada em 28/Jun/2007

Aprovado em 13/Jul/2007 